\title{
Evolution of polymorphism and sympatric speciation through competition in a unimodal distribution of resources
}

\author{
E. Brigatti ${ }^{\dagger \pm}$, J.S. Sá Martins * and I. Roditi ${ }^{\dagger}$ \\ $\dagger$ †entro Brasileiro de Pesquisas Físicas, Rua Dr. Xavier Sigaud 150, 22290-180, Rio de Janeiro, RJ, Brasil \\ *Instituto de Física, Universidade Federal Fluminense, Campus da Praia Vermelha, 24210-340, Niterói, RJ, Brasil \\ 土e-mail address: edgardo@cbpf.br
}

\begin{abstract}
A microscopic agent dynamical model for diploid age-structured populations is used to study evolution of polymorphism and sympatric speciation. The underlying ecology is represented by a unimodal distribution of resources of some width. Competition among individuals is also described by a similar distribution, and its strength is maximum for individuals with the same phenotype and decreases with distance in phenotype space as a gaussian, with some width. These two widths define the model's phase space, in which we identify the regions where an autonomous emergence of stable polymorphism or speciation is more likely.

87.23.Kg, 87.23.-n, 05.10.Ln
\end{abstract}

\section{INTRODUCTION}

The genesis of the incredible diversity of life on our planet is the problem that lies at the heart of the development of modern theory of evolution. This theory attempts to describe all its diverse processes, and in particular speciation, as the outcome of a microscopic dynamics driven by selection and mutation. Insofar as the biological definition of species goes, speciation is the process that explains the generation of two reproductively isolated populations, for which gene flow between the different taxa is absent in any form. The most intuitive conjecture as to how speciation can occur leads to a scenario in which a geographical barrier, or any physical isolation mechanism, separates some fraction of the population of a species. This process is called allopatric speciation. This description is nowadays well accepted and supported by an abundance of empirical evidence $[1,3]$. Much more subtle and complex is the conjecture that speciation is also possible in the absence of any physical isolation mechanism: a single continuous population of interbreeding organisms can split into two reproductively isolated subpopulations. This conjecture is not new, dating at least from Darwin himself [2], albeit from a perspective that is somewhat different from the modern one. A great interest in this theme has grown lately $[4,5]$, strongly supported by observational data related to some fishes of small crater lakes of Cameroon [6] and by recent papers that demonstrate sympatric speciation in vitro [7] or in artificial-life type simulations [8]. On the other hand, the effort towards the construction of general models that can give structure and plausibility to verbal theories (see Ref. [3,9] for a general review) have caused the investigation of an extensive number of mathematical and computational models. Out of the wide variety of such models, we restrict our analysis to ecology-driven reproductive isolation, and we address our attention to models in which natural selection depending on resources distribution originates sympatric speciation. Some of the early attempts on these lines were made with models that represent heterogeneous environments with two niches, and where a mechanism of adaptation to such discrete resources was established. In this case, speciation is caused by a fixed selection that favors phenotypes of both the extremes of the possible range (disruptive selection) and leads to reproductive isolations. A model with these ingredients appeared recently in the literature [10], and a similar one, based on microscopic dynamics, was studied by one of the authors $[15,16]$. The reasoning that underlies these models suggests the question of whether discrete niche might be necessary to explain species coexistence in sympatry. A recent analysis [11] tried to give a negative answer to this question, showing how speciation could arise from competition for continuously distributed resources. In such situations, the environment has just a single ecological niche and, as a consequence, the population feels a stabilizing selection that favors intermediate phenotype. In this scenario, the introduction of a competition mechanism generates a disruptive selection that weakens as species diverge. This competition is generated through a selection force that is stronger for more frequent phenotypes and weaker for rare ones (frequencydependent selection). This mechanism is interesting for two different reasons. First, such a sequence of events is controlled by a frequency-dependent interaction and does not require any externally imposed disruptive selection pressure. On top of that, it appears to be a more general mechanism, which can occur under a much wider range of ecological situations. These two selection pressures are yet not enough to generate speciation. To obtain a stable process, it is necessary to prevent the appearance of intermediate phenotypes through the divergence of reproductive compatibility. This is obtained thanks to the evolution of assortativity, a non-random mating strategy. In the case of a unimodal distribution of resources, such a mechanism is also strictly necessary for the appearance of a first phenotypic divergence. All these ideas have been recently developed in Ref. [11] and are the point of de- 
parture for our present analysis, where we want to study all these results for a more complex microscopic model.

\section{MODEL AND METHODS}

We are interested in working at a microscopic level, where the fundamental agent of the model is an individual of an age-structured population with phenotype variability. For this reason, the classical Penna model [12] is a convenient point of departure, in its asexual haploid and sexual diploid versions. In the following, we will describe results relative to both sexual and asexual taxa. In the first case, we are dealing with the onset of sympatric speciation in a strict sense, which coincides with the setting up of reproductive isolation. In the second, we are investigating the emergence of polymorphism, meaning the splitting of a phenotypical monomorphic population into two (or more) distinct phenotypic clusters. The sexual case describes a diploid population, representing each individual through two sets of coupled genetic strands. Each one is built up by two 32-bit long bit-strings. The first string is age-structured and is used to introduce the biological clock of the individuals, while the other represents the phenotype (trait bit-string). In each of these strings, a gene is encoded by two homologous bits. Its value is 0 if it describes an allele equal to the one present in the original population - wild type allele - or 1 if it has suffered a change and has become a mutant allele. Each individual of a reproductively active couple generates, through a meiotic cycle with crossing-over and recombination, one haploid gamete that, after the introduction of some mutations, combine to form a new genetic strand (see Fig. 1).

The phenotype value is associated with the second string of the genome by the overall sum of the active mutations present in that portion. By active mutation we mean a homozygous locus, where the bit value of the two homologous alleles are equal to one, or a heterozygous one for which the mutant allele is dominant. The number of loci where the mutant alleles are dominant is fixed and their position is chosen randomly at the beginning of the simulation. According to this procedure, the phenotype value $(x)$ is an integer value between 0 and 32 , and is different from the simple sum of all the ones present in the bit-string. This kind of characterization of the phenotype takes into account the diploid nature of the genome and is in some way consistent with the representation of a quantitative trait. This must be understood in the sense that we are representing a trait depending on multiple genes (polygenic trait), but in a particularly simplified situation: the environment has a negligible effect on the phenotype and the quantitative trait is ideal (the genes are randomly associated and their effect is completely additive [14]).

The genetic and, in correspondence, phenotypic variability is assured by the action of mutations. Mutations are obtained by randomly flipping one of the bits of the string. Bits of the trait bit-strings can mutate from 0 to 1 or from 1 to 0 , as opposed to the ageing bit-string that can undergo only bad mutations. This is an oversimplification: for the trait bit-string we do not take into account that it is really difficult for a mutated allele to restore its previous activity. On the contrary, for the ageing bit-string we consider only harmful mutations because of their strong predominance in nature. With the use of this phenotype characterization it is possible to establish a computational representation of intraspecific and/or environmental interaction and/or sexual selection [15-17].

To obtain an age structure in the population, we allow each agent to live until the occurrence of death caused by ageing. This is implemented following rules inspired by Medawar's hypothesis of the accumulation of bad mutations. A position (locus) of the chronological (agestructured) piece of the genome is read at each time step. If an active mutation (defined in the same manner as for the phenotype value) is found at this locus, it is added to the current number of harmful mutations; the individual dies when this amount reaches some pre-determined threshold value.

In the model we introduce another death factor that represents the natural causes of selection in a real ecosystem. It is characterized by three different components. The first is density-dependent, responsible for limiting the number of the total population in accordance with a logistic growth. Then, a frequency-dependent factor takes into account how, in realistic situations, the tendency to occupy the more favored regions in phenotype space is contrasted to an increasing competition between individuals. To these dynamic components of selection, which represent the feedback between individuals and ecosystem and take into account the evolution of the environment, a static component is added. It designs the general ambient condition of the territory and the ecological niche where the agents live. This is a cause of a directional selection that constantly drives the population towards a fitness maximum in phenotype space. To sum up, the general expression for such death factor, as used in the Monte Carlo simulations, is:

$$
V=\frac{\sum_{y=1}^{32} N_{y} \cdot \exp \left(-(x-y)^{2} / 2 c^{2}\right)}{k \cdot \exp \left(-(x-16)^{2} / 2 s^{2}\right)}
$$

At each time step in the simulation a random number is tossed; the individual survives if this number is larger than the $V$ value. The static fitness landscape is represented by a gaussian with deviation $s$ and drives all the population towards the fitness maximum equal to 16. The competition declines with phenotype distance according to a gaussian function with deviation $c$, and the parameter $k$ controls the population dimension. $x$ is the phenotype value of the individual that is feeling the selection pressure and the sum runs over the $y$ index that spans all of the phenotype space. With $N_{y}$ we indicate 
the number of individuals with phenotype $y$.

As already mentioned, positive assortative mating plays a basic role in the path towards speciation. This is obtained by allowing mating only if the distance in phenotype space between two partners is smaller than some predefined threshold $a$ [18]. It is interesting to notice that several models (for example in [11]) implement some mechanism that, by normalizing mating probabilities, guarantees an equal reproductive success for all individuals. This care is due to the fact that assortative mating can cause a sexual selection on rare phenotypes, that have a smaller possibility of finding mates. This byproduct of assortative mating, which has a stabilizing effect that makes speciation less likely [13], can be present in nature. For this reason, in our model, we do not implement any mechanism for equalizing mating success.

Finally, we are not focusing on the problem of the natural evolution of assortativity [11]; in contrast, we are assuming here a fixed assortative rule from the start, for the sake of simplicity. In fact, with such a schematic mechanism, the tuning of the parameter that leads to reproductive isolation is a hard problem.

\section{RESULTS}

We begin the presentation of our results by showing some general data for an asexual population. The dynamical rules for such a population are exactly the same as for the sexual version, except for the fact that we are now dealing with a haploid population, with a very simple reproductive cycle, and where the mutation process stands as the only cause of variability [12].

In this simple case the population evolves rapidly towards the fittest phenotype (16) that corresponds to the maximal carrying capacity. Once this value has been reached, two scenarios are possible. In the first, 16 is a stable point in phenotype space and the population will structure itself in a well shaped gaussian distribution around such value. Otherwise, 16 is still not a fitness maximum (see figure 2), and the population experiences disruptive selection that leads to the appearance of two distinct phenotypic morphs. In this latter case, the tendency to avoid an overcrowded region of phenotype space is stronger than the advantage obtained by reaching the optimal phenotype value. This happens when $c<s$ (see table I). This is not the only possible behavior though; for some small enough values of $c$, the population rapidly splits up its phenotype distribution before its mean reaches the fittest phenotype. An example of this situation is given in the sexual case (see Fig. 6). This particular behavior is related to a stronger influence of competition, that drives a premature bifurcation. In fact, this force increases as $c$ is decreased, as also reflected in Table I by a larger number of occurrences of branching events. This is easily seen, for instance, when $c=10$ where, in just 100000 time steps the population undergoes two bifurcation events, each one causing the appearance of two distinct phenotype clusters, leading towards a three-modal distribution. As we increase the competition further, the population feels such a strong drive that it is impossible to reach a stable polymorphism: the branching events are now so numerous that the phenotype distribution becomes unstable, characterized by a large number of peaks connected by intermediate phenotypes (see inset in figure 2). These results are comparable with the ones obtained in Ref. [11], but the assignment of the phenotype from a genome ruled by microscopic dynamics determines a richer phenomenology. In fact, their prediction of branching for $c<s$ is confirmed by our study, but we were also able to show how branching can occur repeatedly, leading to a polymorphism with more than two phenotype clusters, or to even more complex unstable behavior, as reflected in the enriched unfolding of our model in time. Moreover, data obtained by longer simulation show how the clustering of the population phenotype is biological relevant. In fact, also after $10^{6}$ time steps polymorphism is still present (see figure 3). For this reason, even if from a numerical simulation it is impossible to determine the final equilibrium distribution, such a long-lived structure can not be considered a simple transient phase [19] but, at least, as some sort of long-standing quasi-stationary state with a time scale comparable to the ones of biological interest.

Evolution of diploid sexual populations is marked by a stronger variability, due to the effects of Mendelian segregation and recombination. This fact is a source of difficulties in obtaining sharp phenotypic differentiations because of the constant generation of intermediate phenotypes. For this reason, if the mating process is random, a stable splitting into two different phenotypic clusters can no longer occur, for any possible value of $s$ and $c$. On the contrary, with the introduction of positive assortative mating, not only it is possible to recognize different phenotypic morphs but also speciation with sexually isolated populations (Fig. 4). The parameter space where it occurs appears to be more confined than in the asexual cases. Although still necessary, it is no longer sufficient that $c<s$. Out of the various simulations performed, we have been able to obtain speciation for some simulations with parameters set in the following region:

$$
\begin{aligned}
& s \geq 15,6 \leq c \leq 8, a=4 \\
& s=40, c=6,3 \leq a \leq 5
\end{aligned}
$$

The simulations show that the system is not very sensitive with respect to the value of $s$. If it is sufficiently larger than a particular value of $c$, the population is able to escape from the region with maximum carrying capacity. On the other hand, the value of $c$ can neither be too small, which would lead to a distribution characterized by not well defined connected peaks, nor too large, otherwise the competition would not be strong enough to drive the distribution away from a gaussian centered in 16. Finally, values of $a$ that are too small can to- 
tally trap the variability of the phenotype distribution to a confined region close to 0 or even prevent speciation because of the stronger penalizing effect of sexual selection on rare phenotypes [13]. For larger values, on the opposite, the mating choice is not strict enough to prevent intermediate phenotypes. In any case, even in this more restricted scenario, it is still possible to find some more favorable situations for which, after just 100,000 time steps, two speciation events have already occurred (see figure 6). With respect to the dependence on the initial condition, we remark that if we start with a population having a phenotype close to 16 it is, in general, easier to obtain speciation. We again claim our results to be in accordance with those of Dieckmann and Doebeli [11], but the parameter requirement for the emergency of speciation appears to be more restrictive, even with the usage of a stronger and fixed mating rule. We have also performed some simulations with a model characterized by a big trait bit-string (up to 160 loci) [19]. The fact that no qualitative differences emerged suggests that the results obtained are not dependent on the phenotype range (see Fig. 5).

\section{CONCLUSIONS}

The representation of an individual phenotype character through a second bit-string is a natural extension of the classical Penna model that allows the study of sympatric speciation. Our model was structured under these guidelines and the results corroborate the hypothesis that sympatric speciation emerges from competition for continuously distributed resources. We also used it to test the robustness of this hypothesis under the inclusion of further ingredients and the dependence of the results on the values of some of the model's constitutive variables.

With the study of an age-structured population we can substantiate the claim that the precedent results were not biased by possible long-lived individuals. Moreover, in the asexual population the introduction of a phenotype determined by a genotype with 32 loci confirm previous results [11] in an enriched scenario. The fact that clustering is still present, even after more than $10^{6}$ time steps, determines the biological relevance of such a long-lived phase. For a sexual population where the mechanisms of crossing-over, dominance and homo/heterozygous loci were implemented, the emergence of sympatric speciation is obtained. Our results, obtained with a model that does not include any mechanism for equalizing mating success, contradict the alleged stabilizing effect due to sexual selection caused by assortative mating. Finally, the agility of the Penna model in manipulating bit-strings allowed the run of simulations with large phenotype range, with results that suggest that there is no dependence between speciation and trait range, in spite of a recent claim to the contrary [19]. It is our opinion that agent-based models that allow statistical fluctuations, such as the one in Ref.
[11] and ours, are the most promising testing grounds for evolutionary ideas as a whole.

\section{ACKNOWLEDGMENTS}

We thank the Brazilian agencies CAPES, CNPq, and grants from PRONEX (PRONEX-CNPqFAPERJ/171.168-2003 and PRONEX-FAPERJ E26/171/2003) and FAPERJ (E-26/170.699/2004) for partial financial support.

[1] W.R. Rice,and E.E. Hostert, Evolution 47, 1637 (1993).

[2] C. Darwin, On the origin of species, Cap.4, J. Murray, London (1859).

[3] J.A. Coyne and H. A. Orr, Speciation, Sinauer Associates, Sunderland, MA (2004).

[4] J. Maynard Smith, Am. Nat. 100, 637 (1966).

[5] R. Lande, Proc. Natl. Acad. Sci. USA 78, 3721 (1981).

[6] U.K. Schliewen, D.Tautz and S. Pääbo, Nature 368, 629 (1994).

[7] M.L. Friesen et al., Evolution 58, 254 (2004).

[8] S.S. Chow, C.O. Wilke, C. Ofria, R.E. Lenski and C. Adami, Science 305, 84 (2004).

[9] M. Turelli, N.H. Barton and J.A. Coyne, Trends in Ecol. \& Evol. 16, 330 (2001).

[10] A.S. Kondrashov and F.A. Kondrashov, Nature 400, 351 (1999).

[11] U. Dieckmann and M. Doebeli, Nature 400, 354 (1999).

[12] T.J.P. Penna, J. Stat. Phys. 78, 1629 (1995).

[13] M. Kirkpatrick and S.L. Nuismer, Proc. R. Soc. Lond. B 271, 687 (2004).

[14] D.L. Hartl, A primer of population genetics, Sinauer Associates, Sunderland, MA (1988).

[15] J.S. Sá Martins, S. Moss de Oliveira and G.A. de Medeiros, Phys. Rev. E 64, 021906 (2001).

[16] K. Luz-Burgoa, S. Moss de Oliveira. J.S. Sá Martins, D. Stauffer and A.O. Sousa, Braz. Jour. Phys. 33, 623 (2003).

[17] E. Brigatti, J.S. Sá Martins, I. Roditi, Eur. Phys. J. B 45, 529 (2005).

[18] F. Bagnoli and C. Guardiani, Physica A 347, 534 (2005).

[19] J. Polechova and N.H. Barton, Evolution, 59, 1194 (2005). 


\begin{tabular}{cc|ccccccc}
\hline \hline \multicolumn{1}{c}{$c$} & 40 & 1 & 1 & 1 & 1 & 1 & 1 & 1 \\
& 35 & 1 & 1 & 1 & 1 & 1 & 1 & 2 \\
& 30 & 1 & 1 & 1 & 1 & 1 & 2 & 2 \\
& 25 & 1 & 1 & 1 & 1 & 2 & 2 & 2 \\
& 20 & 1 & 1 & 1 & 2 & 2 & 2 & 2 \\
15 & 1 & 1 & 2 & 3 & 3 & 3 & 2 \\
& 10 & 1 & 3 & 3 & 3 & 3 & 3 & 3 \\
& 5 & $\mathrm{x}$ & $\mathrm{x}$ & $\mathrm{x}$ & $\mathrm{x}$ & $\mathrm{x}$ & $\mathrm{x}$ & $\mathrm{x}$ \\
\hline & & 10 & 15 & 20 & 25 & 30 & 35 & 40 \\
& & & & & & & \\
\hline \hline
\end{tabular}

TABLE I. Generation of polymorphism in an asexual population as we vary the values of the standard deviation of the competition $(c)$ and of the static component $(s)$. Each number in the table corresponds to the number of isolated peaks in the phenotype distribution, while a cross indicates the occurrence of unstable polymorphism, such as the one represented in the inset in figure 2. These results are the outcome of one simulation. We have performed a number of different simulations varying the initial condition. In all cases, the appearance of distinct phenotypic morphs happens only when $c<s$, and the overall dependence of the results on the parameters is similar, even when the number of peaks, isolated or not, vary quite a lot. The simulations run with the same parameters as those of figure 2. The picture was taken after 100, 000 time steps, after all distributions have reached a stationary situation. 


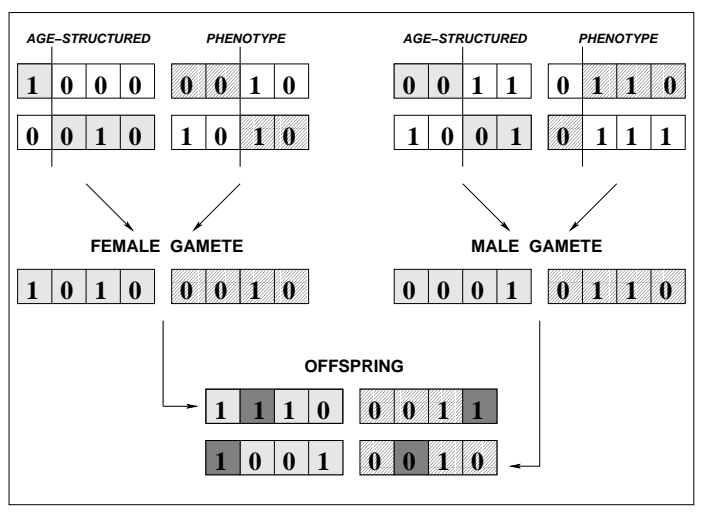

FIG. 1. Example of a reproductive cycle. The diploid genome is represented with its age-structured part (light-shaded background) and the bit-strings that encode for phenotype (diagonal stripes background). After performing crossing-over in the first passage, the haploid gamete is chosen in the second. Finally, some new mutation are added (dark-shaded squares) and the gametes combine to form a new individual.

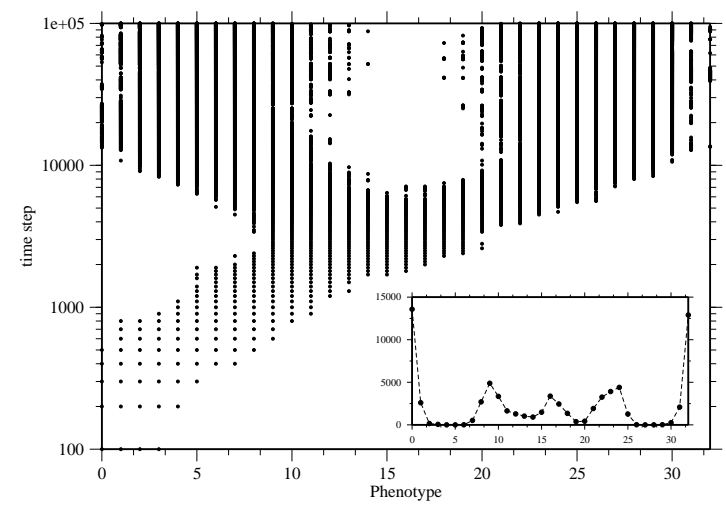

FIG. 2. Time evolution of an asexual population. The parameters used in the simulations are: $k(100,000)$, the initial population $(1,000)$, the minimum reproduction age $(6)$, the maximum reproduction age (32), the number of offspring per mating season (2), the threshold value for harmful diseases (3), the number of mutations added at birth in the age structured string (1), and the mutation probability in the trait string (0.01). The values for the standard deviation of the competition $(c)$ and of the static component $(s)$ are respectively 20 and 25 . The inset shows the unstable distribution generated by a simulation with $s=25$ and $c=5$ after 100,000 time steps.

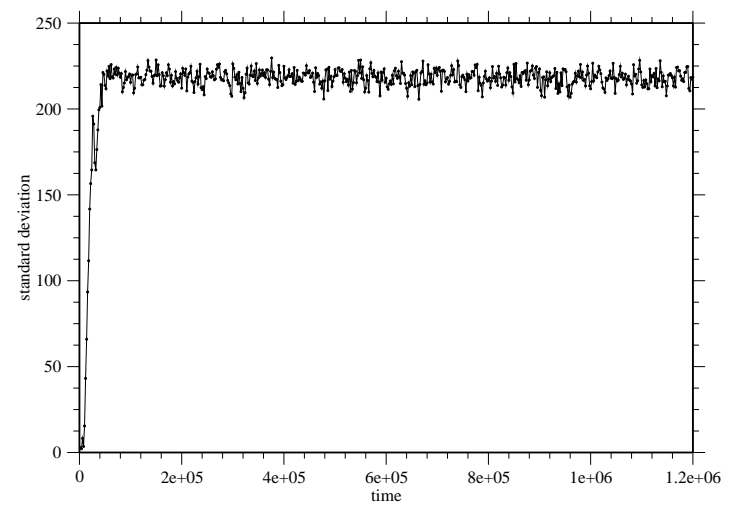

FIG. 3. The standard deviation of the phenotypes distribution can be considered as an order parameter: the splitting of a phenotypical monomorphic population into two (or more) distinct phenotypic clusters corresponds to an abrupt increase in the value of the standard deviation. The fact that after the first transition, near $t=30000$, the mean value of the standard deviation does not change anymore proves the stability of the bimodal phenotypic distribution $(s=25, c=20)$.

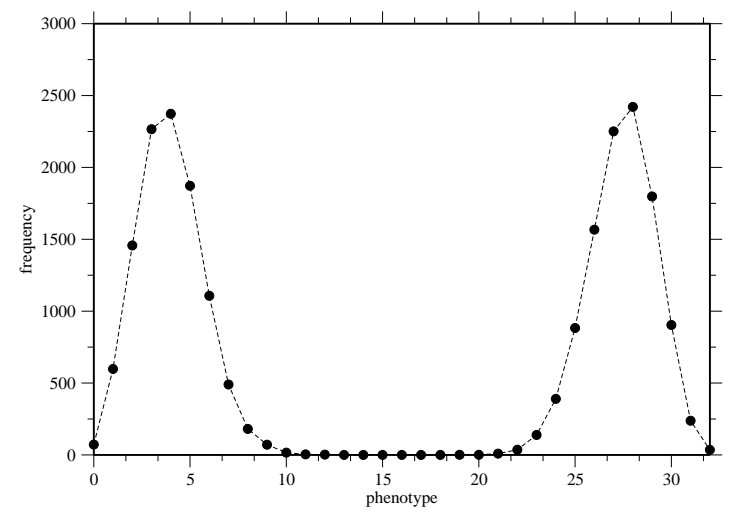

FIG. 4. An example of speciation in a sexual population. The figure shows the phenotype distribution after 100, 000 time steps. The parameters used in the simulations that are different from those of Figure 2 are: $s=40, c=8, a=4$, the minimum reproduction age (8), the number of offspring per mating season (4), and the number of loci where the 1 allele is dominant in each string (16). 


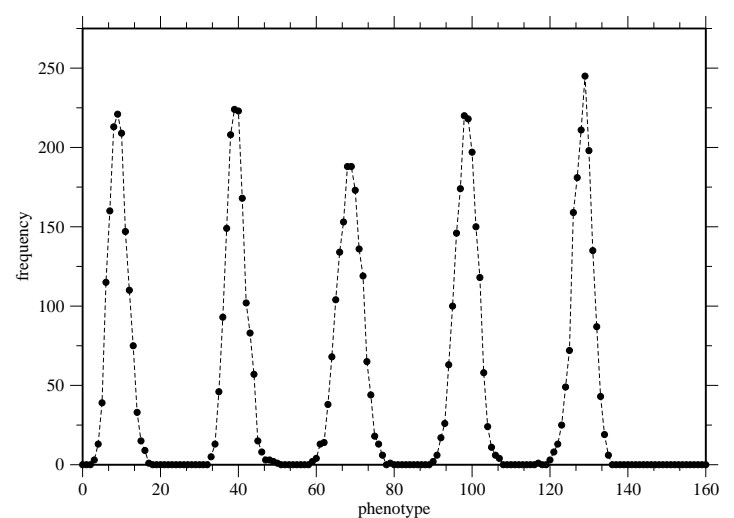

FIG. 5. A simulation with a trait bit-string of 160 loci $(s=160, c=8, a=4)$. This result suggests that there is no dependence between speciation and trait range, in spite of a recent claim to the contrary [19].

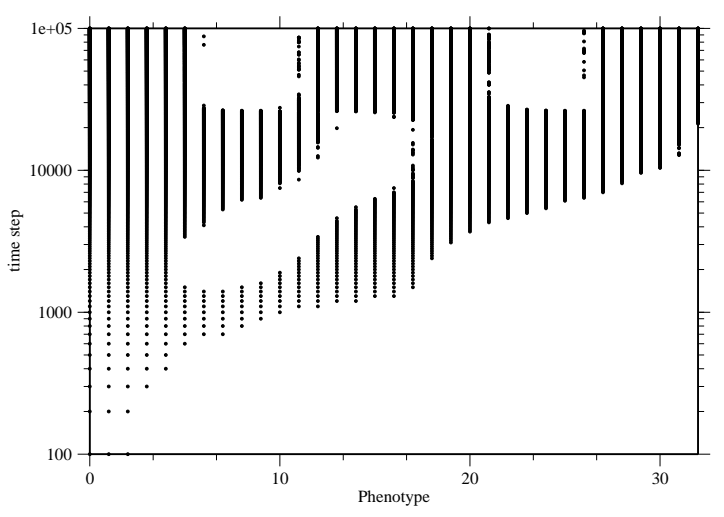

FIG. 6. A particular situation where it is possible to rapidly obtain two speciation events $(s=40, c=6, a=4)$. 